PROCEEDINGS OF THE

AMERICAN MATHEMATICAL SOCIETY

Volume 124, Number 12, December 1996, Pages 3867-3873

S 0002-9939(96)03494-6

\title{
UNIVERSAL CO-ANALYTIC SETS
}

\author{
GREG HJORTH
}

(Communicated by Andreas R. Blass)

\begin{abstract}
There is a universal $\Pi_{1}^{1}$ equivalence relation. The existence of a $\Pi_{1}^{1}$ set universal for $\Pi_{\sim}^{1}$ non-Borel is independent of the usual axioms of mathematics.
\end{abstract}

\section{§0. INTRODUCTION}

One of the fundamental results of descriptive set theory is the existence of a universal co-analytic subset of the plane. Recall that a set $A \subseteq X$, where $X$ is Polish, is said to be analytic, or $\Sigma_{1}^{1}$, if there is a Polish space $Y, B \subseteq Y$ Borel, such that $A$ is a continuous image of $B$. A set $A \subseteq X$ is said to be co-analytic, or $\prod_{1}^{1}$, if $X-A$ is analytic. A Polish space is a separable topological space that admits a complete metric - examples include $\mathbb{R}, \mathbb{C}, \mathbb{R}^{2}, \mathbb{R}^{3},[0,1], 2^{\omega}, \omega^{\omega}$, and so on. A set $A \subseteq \mathbb{R} \times \mathbb{R}$ is universal co-analytic if it is co-analytic and for all $B \subseteq \mathbb{R}, B$ is co-analytic if and only if there exists $z \in \mathbb{R}$ such that

$$
B=A^{z}=_{\mathrm{df}}\{x \in \mathbb{R}:(z, x) \in A\} .
$$

This note investigates the possibility of $\Pi_{1}^{1}$ sets in the plane that are universal for specified subclases of $\Pi_{1}^{1}$. Theorem 1.7 answers a question raised by Kechris at 2.71 of $[\mathrm{Ke}]$ : there is a universal $\Pi_{1}^{1}$ equivalence relation. At 1.1 of [Mi] Miller recalls Mauldin's question regarding the existence of a universal $\Pi_{1}^{1}$ non-Borel set. 2.4 and 2.9 below show that this cannot be decided in ZFC, thereby providing the answer.

The results in this paper will in fact be stated for the Polish space $\omega^{\omega}$. Similar results can be proved for any other perfect Polish space - such as $\mathbb{R}$. Except when there are explicit warnings, the notation below will follow that of [Je]. ZFC* will denote a large, fixed fragment of ZFC. Lower case Greek letters will denote ordinals; lower case Roman letters will denote reals, while uppercase Roman letters will stand for sets of reals. General background can be found in [Je] or [Mo].

\section{$\S 1$. Universal $\Pi_{1}^{1}$ EQUiValence Relations}

In what follows, we will need to not only make use of basic facts about $\Pi_{1}^{1}$ sets such as the existence of norms, as discussed in Chapter 40 of [Je], but also the finer analysis of $\Pi_{1}^{1}$ sets we obtain by considering the effective theory. For instance, if $z \in \omega^{\omega}, L_{\delta}[z]$ admissible, $A \in \Pi_{1}^{1}, \varphi: A \rightarrow \mathrm{WO}$ a $\Pi_{1}^{1}$ norm, where WO is the collection of codes for wellorderings, and $B \subseteq A$ a $\Sigma_{1}^{1}(z)$ set, then the classical

Received by the editors May 2, 1994 and, in revised form, June 12, 1995.

1991 Mathematics Subject Classification. Primary 04A15.

(c)1996 American Mathematical Society 
boundedness theorem tells us that there is some $\alpha<\omega_{1}^{\mathbb{V}}$ such that $|\varphi(x)|$, the order type of $\varphi(x)$, is always less than $\alpha$ for any $x \in A$; a proof of this classical theorem, and a discussion of the definitions involved, can be found in $\S 40$ of [Je]. We will however need the sharper result that there is some $\alpha<\delta$ that provides the bound, and moreover some such $\alpha$ can be produced in $L_{\delta}[z]$ from the codes for $A$ and $B$ in a $\Sigma_{1}^{L_{\delta}[z]}$ fashion; here the reader can find a discussion of the sharper result at $4 \mathrm{~A} .4$ of $[\mathrm{Mo}] ;[\mathrm{Ba}]$ provides a reference for background information regarding admissible sets.

1.1. Definition. $E \subseteq \omega^{\omega} \times \omega^{\omega} \times \omega^{\omega}$ is said to be a universal $\Pi_{1}^{1}$ equivalence relation if:

(i) $E \in \Pi_{1}^{1}$;

(ii) $F \subseteq \omega^{\omega}$ is a $\Pi_{1}^{1}$ equivalence relation if and only if

$$
\exists z \in \omega^{\omega}\left(F=E^{z}={ }_{\mathrm{df}}\{(x, y):(z, x, y) \in E\}\right) .
$$

1.2. Definition. For $E, F \subseteq \omega^{\omega} \times \omega^{\omega}$ both equivalence relations, we write $E \sqsubseteq_{c} F$ if there exists a continuous injection $f: \omega^{\omega} \rightarrow \omega^{\omega}$ such that

$$
\forall x, y \in \omega^{\omega}(x E y \Leftrightarrow f(x) F f(y)) .
$$

For $x \in \omega^{\omega}, \omega_{1}^{x}$ denotes the least $x$-admissible.

1.3 Lemma. If there is a universal $\Pi_{1}^{1}$ equivalence relation, then there is a $\Pi_{1}^{1}$ equivalence relation $E^{*} \subseteq \omega^{\omega}$ such that for any $\Pi_{1}^{1}$ equivalence $F$ we have $F \sqsubseteq_{c} E^{*}$.

Proof. Let $E$ be the universal $\Pi_{1}^{1}$ equivalence relation. Let $\left\langle x_{0}, y_{0}\right\rangle E^{*}\left\langle x_{1}, y_{1}\right\rangle$ if $y_{0}=y_{1}$ and $x_{0} E^{y_{0}} x_{1}$. Then for any $F=E^{z}$, we can let $f(x)=\langle x, z\rangle$ witness the reduction of $F$ to $E^{*}$.

1.4 Definition. Let $A \subseteq \omega^{\omega} \times \omega^{\omega} \times \omega^{\omega}$ be $\Pi_{1}^{1}$, and let $\varphi_{A}: \omega^{\omega} \times \omega^{\omega} \times \omega^{\omega} \rightarrow\{x \in$ $\omega^{\omega}: x$ codes a linear ordering $\}$ arise from the Kleene-Brouwer ordering, so that $\varphi_{A}$ is $\Delta_{1}^{1}$, and $\varphi_{A}^{-1}\left(\left\{x \in \omega^{\omega}: x\right.\right.$ codes a wellordering $\left.\}\right)=A$. Then for $z \in \omega^{\omega}, \alpha \in \omega_{1}$, let $A_{\alpha}^{z}=\left\{\left(y_{0}, y_{1}\right) \in \omega^{\omega} \times \omega^{\omega}:\left(z, y_{0}, y_{1}\right) \in A \wedge \varphi_{A}\left(z, y_{0}, y_{1}\right)<\alpha\right\}$.

1.5 Lemma. Let $\varphi_{A}$ and $A$ be as in 1.4. Suppose $A_{\delta}^{z}$ is an equivalence relation for some $\delta \in \omega_{1}$. Suppose $\gamma$ is z-admissible with $\gamma \leq \delta$. Then:

$$
\left\{\alpha<\gamma: A_{\alpha}^{z} \text { is an equivalence relation }\right\}
$$

is closed and unbounded in $\gamma$.

Proof. Fix $\alpha_{0}<\gamma$. Choose a real $u$ such that $\alpha_{0}$ is recursive in $u$ and $\mathbb{L}_{\gamma}[z, u]$ is admissible; for instance, if we let $u$ arise by generically collapsing $\alpha_{0}$ to $\omega$ over $\mathbb{L}_{\gamma}[z]$, as in 19.9 of [Je], we will still have that $\mathbb{L}_{\gamma}[z, u]$ satisfies $\mathrm{KP}$; an alternative method of finding such a $u$ is provided by [Sa]. Using the $\Sigma_{1}^{1}$ boundedness theorem over $\mathbb{L}_{\gamma}[z, u]$ we can find $\alpha_{1}<\omega_{1}^{(z, u)}, \alpha_{1}>\alpha_{0}$, such that

$$
\begin{aligned}
& \forall x_{0}, x_{1}, x_{2} \in \omega^{\omega} \\
& \left(x_{0}, x_{0}\right) \in A_{\alpha_{1}}^{z}, \\
& \left(x_{0}, x_{1}\right) \in A_{\alpha_{0}}^{z} \Rightarrow\left(x_{1}, x_{0}\right) \in A_{\alpha_{1}}^{z}, \\
& \left(x_{0}, x_{1}\right) \in A_{\alpha_{0}}^{z},\left(x_{1}, x_{2}\right) \in A_{\alpha_{0}}^{z} \Rightarrow\left(x_{0}, x_{2}\right) \in A_{\alpha_{1}}^{z} .
\end{aligned}
$$


The existence of such an $\alpha_{1}$, working for all $x_{0}, x_{1}, x_{2}$ in $\mathbb{V}$, follows as in the remarks preceding 1.1. Repeating the argument, and using the $\Sigma_{1}$ definability of $\alpha_{i+1}$ from $\alpha_{i}$, we obtain a sequence $\left(\alpha_{i}\right)_{i \in \omega} \in \mathbb{L}_{\gamma}[z, u]$ such that for all $i \in \omega, \forall x_{0}, x_{1}, x_{2} \in \omega^{\omega}$

$$
\begin{aligned}
& \alpha_{i}<\alpha_{i+1}, \\
& \left(x_{0}, x_{1}\right) \in A_{\alpha_{i}}^{z} \Rightarrow\left(x_{1}, x_{0}\right) \in A_{\alpha_{i+1}}^{z}, \\
& \left(x_{0}, x_{1}\right) \in A_{\alpha_{i}}^{z},\left(x_{1}, x_{2}\right) \in A_{\alpha_{i}}^{z} \Rightarrow\left(x_{0}, x_{2}\right) \in A_{\alpha_{i+1}}^{z} .
\end{aligned}
$$

Then $\alpha=\bigcup_{i \in \omega} \alpha_{i}<\gamma$ is as required to show unboundedness.

The fact that $\left\{\alpha<\gamma: A_{\alpha}^{z}\right.$ is an equivalence relation $\}$ is closed follows from the well-known observation that an increasing union of equivalence relations again forms an equivalence relation.

1.6 Lemma. Let $\varphi_{A}$ and $A$ be as in 1.4. Then for $z \in \omega^{\omega},\left\{\alpha \in \omega_{1}: A_{\alpha}^{z}\right.$ is an equivalence relation $\}$ is $\Pi_{1}^{1}(z)$ in the codes.

Proof. $A_{\alpha}^{z}$ is uniformly $\Delta_{1}^{1}(z, \alpha)$, and thus the statement that it forms an equivalence relation is uniformly $\Pi_{1}^{1}(z, \alpha)$.

1.7 Theorem. There exists a universal $\Pi_{1}^{1}$ equivalence relation.

Proof. Let $G \subseteq \omega^{\omega} \times \omega^{\omega} \times \omega^{\omega}$ be universal $\Pi_{1}^{1}$, so that $B \subseteq \omega^{\omega} \times \omega^{\omega}$ is $\Pi_{1}^{1}$ if and only if there exists $z \in \omega^{\omega}$ such that $B=G^{z}={ }_{\mathrm{df}}\{(x, y):(z, x, y) \in G\}$. Let $A=G \cup\left\{(z, x, x): z, x \in \omega^{\omega}\right\}$, so that $A^{z}$ is the minimal reflexive relation including $G^{z}$ for all $z \in \omega^{\omega}$. Fix $\varphi_{A}$ for $A$ as in 1.4. Without loss of generality $\varphi_{A}(z, x, x)=0$ for all $x, z$.

Now let $E=\left\{(z, x, y) \in A: \exists \alpha<\omega_{1}^{(x, y, z)}\right.$ such that $A_{\alpha}^{z}$ is an equivalence relation, $\left.\varphi_{A}(z, x, y)<\alpha\right\}$.

Claim. $E$ is $\Pi_{1}^{1}$.

This follows at once from Spector-Gandy in light of 1.6; further details can be found in $4 \mathrm{~F}$ of $[\mathrm{Mo}]$.

$(\square$ claim)

Claim. If $G^{z}$ is an equivalence relation, then $E^{z}=G^{z}$.

This follows at once from the definitions and 1.5.

$(\square$ claim $)$

Claim. $\forall z \in \omega^{\omega}\left(E^{z}\right.$ is an equivalence relation $)$.

Since $E^{z}=\bigcup\left\{A_{\alpha}^{z}: \alpha \in \omega_{1}, A_{\alpha}^{z}\right.$ is an equivalence relation $\}$ by 1.5. ( $\square$ claim)

The last three claims complete the proof. Thus, as a consequence of 1.3 , we obtain a $\Pi_{1}^{1}$ equivalence relation $F$ such that if $E$ is any $\Pi_{1}^{1}$ equivalence relation, then $E \sqsubseteq_{c} F$.

\section{§2. Universal $\Pi_{1}^{1}$ NON-BOREL}

2.1 Definition. $A \subseteq \omega^{\omega} \times \omega^{\omega}$ is said to be universal for $\Pi_{1}^{1}$ non-Borel if:

(i) $A \in \Pi_{1}^{1}$;

(ii) $B \subseteq \omega^{\omega}$ is $\prod_{1}^{1}$ and non-Borel if and only if $\exists z \in \omega^{\omega}$ such that

$$
B=A^{z}=_{d f}\left\{x \in \omega^{\omega}:(z, x) \in A\right\} .
$$

2.2 Definition. $A \subseteq \omega^{\omega} \times \omega^{\omega}$ is said to be universal for uncountable $\Sigma_{2}^{1}$ if:

(i) $A \in \Sigma_{2}^{1}$; 
(ii) $B \subseteq \omega^{\omega}$ is an uncountable $\sum_{2}^{1}$ set if and only if $\exists z \in \omega^{\omega}$ such that

$$
B=A^{z} .
$$

2.3 Lemma. If $\mathbb{V}=\mathbb{L}$, then there is no universal for uncountable $\Sigma_{2}^{1}$ set.

Proof. Suppose then that we have $\Phi: \omega_{1} \rightarrow \omega^{\omega} \Delta_{2}^{1}$ in the codes and $A \subseteq \omega^{\omega} \Sigma_{2}^{1}$ with only uncountable sections. It suffices to find $B \in \Sigma_{2}^{1}$ such that $B \subseteq \omega^{\omega}, B \notin$ $\left\{A^{z}: z \in \omega^{\omega}\right\}$ and $B$ uncountable.

We now define two functions with domain $\omega_{1}$ by simultaneous induction on $\alpha \in \omega_{1}$. Given $g(\beta)$ defined for all $\beta \leq \alpha$, we let $f(\alpha)$ be the least $\gamma \in \omega_{1}$ such that for $w=\Phi(\gamma)$ we have $w \notin \mathbb{L}_{g(\alpha)}$. We then let $g(\alpha+1)$ be the least $\delta$ such that

(i) $\mathbb{L}_{\delta} \vDash \mathrm{ZFC}^{*}, \delta>\alpha, \delta>\sup \{g(\beta): \beta \leq \alpha\}$;

(ii) $\exists x, y \in \mathbb{L}_{\delta}\left(\Phi(\alpha)=x \wedge \forall \beta \leq \alpha(y \neq \Phi(f(\beta))) \wedge y \in A^{x}\right)$.

For $\lambda$ a limit we set $g(\lambda)=\bigcup_{\alpha \in \lambda} g(\alpha)$.

It follows from the construction that $f$ and $g$ are $\Sigma_{2}^{1}$ in the codes. Thus $B=$ $\left\{w \in \omega^{\omega}: \exists \beta \in \omega_{1}(w=\Phi(f(\beta)))\right\}$ is $\Sigma_{2}^{1}$, and is uncountable by the assumptions that $\Phi$ has uncountable image and $A$ has uncountable sections. Moreover, it also follows from $\Phi$ being a surjection that $B \notin\left\{A^{z}: z \in \omega^{\omega}\right\}$.

2.4 Theorem. If $\mathbb{V}=\mathbb{L}$, then there is no universal for $\Pi_{1}^{1}$ non-Borel set.

Proof. Suppose instead that $A \subseteq \omega^{\omega} \times \omega^{\omega} \times \omega^{\omega}$ is $\Pi_{1}^{1}$ and for $B \subseteq \omega^{\omega} \times \omega^{\omega}$ an arbitrary set in the plane, $B \in \Pi_{1}^{1}-\Delta_{1}^{1}$ if and only if $\exists x \in \omega^{\omega}$ such that $B=A^{x}$. Using some $\Delta_{1}^{1}$ pairing function $\langle\cdot, \cdot\rangle: \omega^{\omega} \cong \omega^{\omega} \times \omega^{\omega}$, this is seen to be equivalent to the existence of a set as in 2.1. It will suffice to demonstrate a contradiction by producing a universal for uncountable $\Sigma_{2}^{1}$ set.

Let $A_{0} \subseteq A$ uniformize $A$ in the third coordinate, so that $A_{0}$ is $\Pi_{1}^{1}$ and

$$
\forall x, y \in \omega^{\omega}\left(\exists z((x, y, z) \in A) \Leftrightarrow \exists ! z\left((x, y, z) \in A_{0}\right)\right) .
$$

Let $B \subseteq \omega^{\omega} \times \omega^{\omega}$ be given by $\left.B=\{(x, y): \exists z(x, y, z) \in A)\right\} \bigcup\{(x, y)$ : $\left.\exists z_{0}, z_{1}\left(\left(x, z_{0}, y\right) \in A_{1}\left(x, z_{0}, z_{1}\right) \in A_{0}, z_{1} \neq y\right)\right\}$. $B$ is $\Sigma_{2}^{1}$. Moreover, if $x \in \omega^{\omega}$, then $A^{x}$ is non-Borel, hence uncountable, and thus $B^{x}=\{y:(x, y) \in B\}$ is again uncountable.

Let $G$ be an uncountable $\Sigma_{2}^{1}$ set. Say $G=\left\{y: \exists z(y, z) \in G_{0}\right\}$ where $G_{0}$ is $\prod_{1}^{1}$. Let $G_{1}=\left\{(y, z): z\right.$ codes a wellfounded model, $\mathbb{L}_{\alpha} \vDash \mathrm{ZFC}^{*}, y \in \mathbb{L}_{\alpha}, \mathbb{L} \alpha \vDash$ " $\exists y_{0}$ such that $\left(y, y_{0}\right) \in G_{0}$ " $\}$. Now let $G_{2} \subseteq G_{1}$ uniformize $G_{1}$ in the second coordinate with $G_{2} \in \prod_{1}^{1}$.

It follows from the definitions and $\mathbb{V}=\mathbb{L}$ that $G=\left\{y: \exists z(y, z) \in G_{2}\right\}$. Since $\mathbb{V}=\mathbb{L}$ and $G$ is uncountable, $G_{2}$ is non-Borel. By assumption on $A$ we can find $x$ such that $G_{2}=A^{x}$. But now since $G_{2}$ has been uniformized in the second coordinate $A^{x}=A_{0}^{x}$, and $B^{x}=G_{2}$, as required.

It will be convenient, during the remainder of the paper, for us to make liberal use of the definitions and theorems from forcing. For instance, if $M$ is a structure, $\mathbb{P}$ is a partial order in $M, \varphi$ a sentence in the language of set theory, then the display

$$
M^{\mathbb{P}} \models \varphi
$$

is simply a shorthand expression for saying that whenever $G \subseteq \mathbb{P}$ is $M$-generic, $G$ appearing in a generic extension of $\mathbb{V}$, then $M[G] \models \varphi$. It is a standard fact 
that if $\varphi(\dot{x})$ is $\Sigma_{n}$ in the Levy hierarchy, and $M$ satisfies $\Delta_{0}$-comprehension and $\Sigma_{n}$-replacement, then the set of $a \in M$ such that

$$
M^{\mathbb{P}} \models \varphi(a)
$$

is uniformly $\Sigma_{n}(\mathbb{P})$ over $M$.

Chapter 3 of [Je] provides an excellent source for these and other basic facts about forcing. There is one respect in which I will go beyond the notation of [Je], and that is in using $\operatorname{Coll}(\omega, \alpha)$ to denote the forcing notion that collapses $\alpha$ to $\omega$; this partial order, consisting of finite partial functions from $\omega$ to $\alpha$, is discussed at 19.9 of [Je].

It is worth noting in advance that if $A \in \Pi_{1}^{1}(x)$, then there is a sense in which the issue of whether $A$ is Borel is internal to $\mathbb{L}[x]$. Note that $A \in \Delta_{1}^{1}$ if and only if there is some $\alpha<\omega_{1}^{\mathbb{V}}$ such that for $\varphi_{A}: A \rightarrow$ WO a $\Pi_{1}^{1}(x)$ norm on $A, \varphi_{A}(y)$ has order type less than $\alpha$ for all $y \in A$. However, this statement is $\Pi_{2}^{1}(x, \alpha)$, and hence absolute to $\mathbb{L}[x]^{\operatorname{Coll}(\omega, \alpha)}$. Therefore, we can determine whether $A$ is Borel in $\mathbb{V}$ by asking whether $A$ is Borel in a suitably chosen generic extension of $\mathbb{L}[x]$.

We will also be making use of the notion of sharps. This large cardinal hypothesis states that for every $x \in \omega^{\omega}$ there exists a corresponding $x^{\#}$. This $x^{\#}$ codes a theory of indiscernibles for $\mathbb{L}[x]$. Provided we choose an appropriate coding system, we may assume $x^{\#} \in \omega^{\omega}$. $x^{\#}$ will be a $\Pi_{2}^{1}(x)$ singleton, and satisfy the key conditions of wellfoundedness and remarkability - as mentioned following 2.5 below. It is perhaps worth remarking that the existence of $x^{\#}$ implies the existence of a club class of indiscernibles for $\mathbb{L}[x]$; moreover, we will have that for any $n \in \omega, \varphi$ a formula in set theory, $\mathbb{L}[x] \models \varphi\left(x, \omega_{1}^{\mathbb{V}}, \cdots, \omega_{n}^{\mathbb{V}}\right)$ if and only if $x^{\#}$ codes the formula asserting that $\varphi\left(x, c_{1} \cdots c_{n}\right)$ holds, where $c_{1}, \cdots, c_{n}$ are names for the first $n$ many indiscernibles. The reader can find a more complete discussion of sharps in $\S 30$ of [Je].

In order to complete the proof of the complementary result for 2.4 it will be necessary to introduce some unpleasant notation. Before giving this notation, it might be helpful to briefly describe the ideas that will underlie the proof. Let us start to assume $\forall x \in \omega^{\omega}\left(x^{\#} \exists\right)$.

We should begin by thinking of $\Pi_{1}^{1}$ sets as built up effectively in $\omega_{1}$ many "stages" - just as in recursion theory we may think of a recursively enumerable set as being a countable union of recursive sets. Now fix $A_{0}$ universal $\Pi_{1}^{1}$. We describe the sections of our universal $\Pi_{1}^{1}$ non-Borel set $A$. Take $z \in \omega^{\omega}$, and view it as a "guess" at $x^{\#}$, for some $x \in \omega^{\omega}$, where $\mathbb{L}[x]$ calculates that $A_{0}^{x}$ is non-Borel. As long as we are at a stage where the guess is good, we place the reals in $A_{0}^{x}$ into $A^{z}$; as soon as the guess starts going bad, we add in the reals of some fixed $\Pi_{1}^{1}$ non-Borel set $G$. If this is all arranged correctly, then we will have $A^{z}$ non-Borel for all $z \in \omega^{\omega}$, and for $z=x^{\#}, A_{0}^{x}$ non-Borel, we will have $A^{z}=A_{0}^{x}$.

2.5 Notation. For $z$ a theory of indiscernibles with definable Skolem functions, $\eta$ a linear ordering, let $\Gamma(z, \eta)$ denote the model generated by applying $z$ to the indiscernibles $\left(c_{i}\right)_{i \in \eta}$ and closing under the Sholem functions. (This is what $\S 30$ of [Je] would refer to as $(\Sigma, \eta)$, for $\Sigma=z$.)

As mentioned above, two crucial properties of $x^{\#}$ are remarkability and wellfoundedness. Remarkability asserts that $\Gamma\left(x^{\#}, \bar{\eta}\right)$ end extends $\Gamma\left(x^{\#}, \eta\right)$ whenever $\eta$ has limit type and is an initial segment of $\bar{\eta}$. Wellfoundedness asserts that $\Gamma\left(x^{\#}, \alpha\right)$ 
is wellfounded for any ordinal $\alpha$. Remarkability is essentially a syntactical requirement on $x^{\#}$, while wellfoundedness is $\Pi_{2}^{1}(x)$. By the earlier remarks, for $A \in \Pi_{1}^{1}(x)$, assuming $x^{\#}, A$ will be Borel if and only if

$$
\mathbb{L}[x]^{\operatorname{Coll}\left(\omega,<\omega_{1}^{\mathrm{V}}\right)} \models \text { "A Borel" }
$$

if and only if

$$
\mathbb{L}[x]^{\operatorname{Coll}\left(\omega, \omega_{1}^{\mathbb{V}}\right)} \models " A \text { Borel, } "
$$

since $\mathbb{L}_{\omega_{1}^{\mathrm{v}}}(x) \prec \mathbb{L}[x]$; this is in turn equivalent to $x^{\#}$ coding the formula asserting that $\mathbb{L}[x]^{\operatorname{Coll}\left(\omega, c_{1}\right)}$ satisfies that $A$ is $\Delta_{1}^{1}$.

2.6 Notation. For the rest of the paper, fix $A_{0} \subseteq \omega^{\omega}$ universal $\Pi_{1}^{1}$ in the usual sense, so that $B \subseteq \omega^{\omega}$ is $\prod_{1}^{1}$ if and only if $\exists z \in \omega^{\omega}\left(B=A_{0}^{z}\right)$. Let $\varphi_{A_{0}}$ be a $\Delta_{1}^{1}$ norm for $A_{0}$, so that $\forall x_{0}, x_{1} \in \omega^{\omega}\left(\left(x_{0}, x_{1}\right) \in A_{0} \Leftrightarrow \varphi_{A_{0}}\left(x_{0}, x_{1}\right) \in \mathrm{WO}\right)$. Fix $G \subseteq 2^{\omega} \Pi_{1}^{1}$ and non-Borel. Fix $\varphi_{G}$ a $\Delta_{1}^{1}$ norm for $G$.

2.7 Notation. Let $D \subseteq \omega^{\omega}$ consist of those reals $z \in \omega^{\omega}$, such that for some real $x(z) \in \omega^{\omega}$ we have:

(i) $z$ codes a theory of indiscernibles for $\mathbb{L}[x(z)]$, and $z$ satisfies the first order requirements of remarkability and indiscernibility;

(ii) $\Gamma(z, \omega)$ is an $\omega$-model;

(iii) $x(z)=\left\langle x_{0}(z), x_{1}(z)\right\rangle$, where $x_{0}(z)$ codes a pair of continuous functions $f_{0}, f_{1}$ : $2^{\omega} \rightarrow \omega^{\omega}$, such that for all $y \in 2^{\omega}, f_{1}(y)$ witnesses that $f_{0}(y) \notin A_{0}^{x_{1}(z)}$, and such that $f_{0}$ is injective;

(iv) $\Gamma(z, \omega) \vDash " A_{0}^{x_{1}(z)}$ is non-Borel in $\mathbb{L}[x(z)]^{\operatorname{Coll}\left(\omega, c_{1}\right)}$."

It is routine to check that $D$ is $\Delta_{1}^{1}$ : As remarked earlier, (i) is esentially syntactical; (ii) is Borel, since $\Gamma(z, \omega)$ can be recovered from $z$ in a Borel fashion; that (iii) is Borel follows from the compactness of $2^{\omega}$ and the fact that $x(z)$ can be produced in a uniformly $\Delta_{1}^{1}(z)$ manner; and finally, (iv) is simply a statement about the theory coded by $z$, and is therefore arithmetical in $z$. As in the remarks following 2.5 , if $z$ satisfies (iv) and $z=x(z)^{\#}$, then we will indeed have that $A_{0}^{x_{1}(z)}$ is non-Borel. Note that for $z \in D$ it follows from (ii) and the requirement of remarkability that $\Gamma(z, \eta)$ is an $\omega$-model for any linear ordering $\eta$.

2.8 Lemma. For $z \in D, z=x(z)^{\#}$ if and only if for all $\alpha<\omega_{1}$ the wellfounded part of $\Gamma(z, \alpha+\omega)$ includes $\alpha$.

Proof. The condition on the right-hand side is necessary since $\Gamma\left(x(z)^{\#}, \alpha+\omega\right) \supseteq \alpha$. That it is sufficient follows by remarkability.

Note that if $\alpha$ is not included in the wellfounded part of $\Gamma(z, \alpha+\omega)$, then, by remarkability, $\alpha$ is not included in the wellfounded part of $\Gamma(z, \beta+\omega)$ for any $\beta>\alpha$.

2.9 Theorem. If $\forall x \in \omega^{\omega}\left(x^{\#} \exists\right)$, then there is a universal $\Pi_{1}^{1}$ non-Borel set.

Proof. We describe $A \subseteq \omega^{\omega} \times \omega^{\omega}$ fiber by fiber. Fix $z \in \omega^{\omega}$. First of all, if $z \notin D$, then let $A^{z}=i(G)$, where $i: 2^{\omega} \rightarrow \omega^{\omega}$ is the natural injection.

So now suppose $z \in D$. Let $x=\left\langle x_{0}, x_{1}\right\rangle=x(z)$ where $x_{0}$ codes $f_{0}, f_{1}$ as above. Let $A^{z}=\left\{y \in \omega^{\omega}: y \in A_{0}^{x_{1}}\right.$, and for $\left\|\varphi_{A_{0}}\left(x_{1}, y\right)\right\|=\alpha, \Gamma(z, \alpha+\omega)$ includes $\alpha\} \cup\left\{y \in \omega^{\omega}: \exists w \in 2^{\omega}, y=f_{0}(w), w \in G\right.$, and for $\left\|\varphi_{G}(w)\right\|=\alpha, \Gamma(z, \alpha+\omega)$ does not include $\alpha\}$. 
Claim. $\forall z \in \omega^{\omega}\left(A^{z}\right.$ is $\left.\prod_{1}^{1}\right)$.

This is immediate if $z \notin D$, so suppose instead that $z \in D$. Then the calculation of $f_{0}^{-1}(y)$, assuming it exists, is uniformily $\Delta_{1}^{1}(z, y)$, since $f_{0}$ is injective. For $v \in$ WO, the calculation $\Gamma(z,\|v\|+\omega) \supseteq\|v\|$ can be phrased in both a $\Sigma_{1}^{1}(z, v)$ and a $\Pi_{1}^{1}(z, v)$ fashion, thus showing that $\left\{y \in A_{0}^{x_{1}}:\left\|\varphi_{A_{0}}\left(x_{1}, y\right)\right\|=\alpha \Rightarrow \Gamma(z, \alpha+\omega) \supseteq\right.$ $\alpha\}$ and $\left\{w \in G:\left\|\varphi_{G}(w)\right\|=\alpha \Rightarrow \Gamma(z, \alpha+\omega) \nsupseteq \alpha\right\}$ are uniformily $\Pi_{1}^{1}(z)$. Since $f_{0}^{\prime \prime}\left[2^{\omega}\right]$ is compact, and $\Delta_{1}^{1}\left(x_{0}\right)$, this suffices.

( $\square$ claim)

Claim. $A$ is $\Pi_{1}^{1}$.

By the uniformity of the definition of $A^{z}$ as $z$ ranges over $D$ and $\sim D$.( $\square$ claim)

Claim. $\forall z \in \omega^{\omega}\left(A^{z}\right.$ is non-Borel $)$.

Again this is immediate if $z \notin D$, so suppose $z \in D$. Now if we in fact have $x^{\#}=z$, then there will be arbitrarily large $\gamma<\omega_{1}^{\mathbb{V}}$ such that $\mathbb{L}[x]^{\operatorname{Coll}(\omega, \gamma)} \vDash " \exists y \in$ $A_{0}^{x_{1}}\left(\varphi_{A_{0}}\left(x_{1}, y\right) \geq \gamma\right)$ ", by condition (iv) of 2.7 , and thus $A_{0}^{x_{1}}$ must be non-Borel; since $A_{0}^{x_{1}}=A^{z}$ by construction, this suffices.

So, suppose instead that $z \in D, z \neq x^{\#}$, and $\alpha$ is least so that $\Gamma(z, \alpha+\omega) \nsupseteq \alpha$ (the existence of such an $\alpha$ follows by 2.8); note then that $\forall \beta>\alpha \Gamma(z, \beta+\omega) \nsupseteq \alpha$. Therefore $A^{z}$ consists of the disjoint union of $\left\{y \in \omega^{\omega}: y \in A_{0}^{x_{1}},\left\|\varphi_{A_{0}}\left(x_{1}, y\right)\right\|<\alpha\right\}$ and $\left\{f_{0}(w): w \in G\left\|\varphi_{G}(w)\right\| \geq \alpha\right\}$. Since $f_{0}^{\prime \prime}\left[2^{\omega}\right]$ is disjoint from $\left\{y \in \omega^{\omega}: y \in\right.$ $\left.A_{0}^{x_{1}},\left\|\varphi_{A_{0}}\left(x_{1}, y\right)\right\|<\alpha\right\}$ and since $\left\{w \in G:\left\|\varphi_{G}(w)\right\| \geq \alpha\right\}$ is non-Borel, it follows that $A^{z}$ is non-Borel, as required.

Claim. If $B \subseteq \omega^{\omega}$ is $\prod_{1}^{1}$ non-Borel, then $\exists z \in \omega^{\omega}\left(A^{z}=B\right)$.

Since $B$ is not $\Delta_{1}^{1}, \sim B$ is uncountable, and thus contains a perfect set. Let $\left.B=\left\{y \in \omega^{\omega}: \forall x \in \omega^{\omega}(y, x) \notin B_{0}\right)\right\}$ where $B_{0}$ is closed, and fix $f_{0}, f_{1}: 2^{\omega} \rightarrow \omega^{\omega}$ uniformly continuous with $\left(f_{0}(w), f_{1}(w)\right) \in B_{0}$ for all $w \in 2^{\omega}$. Let $x_{0}$ code $\left(f_{0}, f_{1}\right)$. Choose $x_{1}$ so that $B=A_{0}^{x_{1}}$. Let $x=\left\langle x_{0}, x_{1}\right\rangle$. It is immediate from the definitions that for $z=x^{\#}$ we have $A^{z}=B$.

$(\square$ claim)

The last three claims complete our argument by ensuring that $A_{1}$ will be our universal $\Pi_{1}^{1}$ non-Borel set.

\section{REFERENCES}

[Ba] J. Barwise, Admissible sets and structures, Springer-Verlag, New York, 1975. MR 54:12519 [Je] T. Jech, Set theory, Academic Press, San Diego, 1978. MR 80a:03062

[Ke] A. S. Kechris, Lectures on definable group actions and equivalence relations, Unpublished manuscript.

[Mi] A. W. Miller, Arnie Miller's problem list, Set theory of the reals (H. Judah, ed.), IMCP, Bar-Ilan, 1993. MR 94m:03073

[Mo] Y. N. Moschovakis, Descriptive set theory, North-Holland Publishing Company, Amsterdam, New York, Oxford, 1980. MR 82e:03002

[Sa] G. E. Sacks, Countable admissible ordinals and hyperdegrees, Advances in Mathematics 19 (1976), 213-262. MR 55:2536

Department of Mathematics, California Institute of Technology, Pasadena, CaliFORNIA 91125

Current address: Department of Mathematics, University of California, Los Angeles, California 90024-1555

E-mail address: greg@cco.caltech.edu

E-mail address: greg@math.ucla.edu 